\title{
Hypothèses au sujet de l'adaptation des Amibes du groupe limax à l'homme et aux animaux
}

\author{
par J. B. JADIN \\ Département de Protozoologie. \\ Insitut de Médecine tropicale «Prince Léopold» (Directeur: Pr P. G. JANssEns) \\ Nationalestraat 155, 2000 Anvers, Belgique
}

\section{Résumé}

Les auteurs envisagent diverses hypothèses qui font entrevoir le mécanisme de la virulence des souches d'amibes de l'eau. Il est vraisemblable que c'est le passage intensifié des amibes d'un sujet à l'autre par le surpeuplement des piscines qui est à la base de cette virulence.

L'existence d'une souche de Naegleria gruberi pathogène distincte de celles retrouvées habituellement dans l'eau des bassins est possible.

\section{Summary}

The authors consider different hypothesises which more or less explain the virulence mecanism of some strains of water amebae.

It is most likely that the intensive passage of amebae from one human to another, in overcrowded swimming pools, explains this virulence.

Existence of a pathogenic strain of Naegleria gruberi, distinct of those usually found in swimming pool water, is quite possible. 
Les recherches de Culbertson et de ses collaborateurs (1959) ont montré que des amibes du groupe Limax étaient capables de se développer dans le cerveau des singes et des souris. Cette découverte a ouvert la voie à la connaissance de la méningoencéphalite amibienne. A la suite de Fowler et Carter (1965), les chercheurs ont décrit dans une cinquantaine de cas la présence de Naegleria gruberi et d'Hartmannella dans le cerveau de l'homme.

En réalité, quand on reprend les travaux des auteurs du début de ce siècle et plus particulièrement l'étude de Wells (1911) et celle de Chatton - Lalung-Bonnaire (1912), il faut bien admettre que les amibes libres, du groupe Limax, ont été retrouvées dès le début de ce sièzle dans l'intestin de l'homme et parfois dans les abcès du foie. Rappelons qu'à la suite de Schaudinn (1903) et de Kartulis (1904), tous les abcès amibiens du cerveau avaient été attribués à Entamoeba histolytica sans que cette amibe ait été mise en évidence pour chaque cas.

Parmi les 61 cas colligés par Halpert et Ashley (1944), cinq seulement apparaissent comme des métastases directes d'origine intestinale. Dans son traité sur l'amibe dysentérique, R. Deschiens (1965) constate judicieusement qu'il est difficile d'expliquer pourquoi ces abzès du cerveau surviennent quand les lésions antérieures du foie ou du poumon ou de l'intestin sont en voie de cicatrisation ou complètement cicatrisées.

Le pouvoir pathogène des amibes du groupe Limax étant actuellement bien établi et connaissant leur énorme dispersion dans l'eau et le sol humide, il est légitime de se demander comment un organisme ausi répandu devient tout à coup pathogène au point d'entraîner la mort en 3 ou 4 jours.

C'est à cette question que nous voudrions tenter de répondre en envisageant diverses possibilités.

\section{$1^{\circ}$ Pouvoir pathogène d'emblée.}

Les deux souches de Naegleria gruberi 0359 et 0560 que nous avons isolé (Jadin et coll. 1971) à partir de deux cas de méningo-encéphalite amibienne mortelle ont tué les souris inoculées dans le cerveau à partir du liquide céphalo-rachidien après 48 heures. La souche 0359 a été retransmise à 100 reprises par voie intracérébrale chez la souris avec une virulence inchangée. Ces souches entretenues cinquante fois en milieu liquide à la casitone de Cervà (1969) gardent leur virulence pour la souris. Il en est de même si on les conserve à $-196^{\circ} \mathrm{C}$ en azote liquide.

Les souches de Naegleria gruberi Vitek de Cervà (Cervà et coll. 1969) et HB 1 de Butt (Butt et coll. 1968 et Culbertson et coll. 1968) ont un comportement comparable.

Il est donc évident que les souches d'amibes isolées à partir de cas de méningoencéphalite sont pathogènes et le restent.

La répétition des 16 cas mortels observés à Usti (Cervà et coll. 1968) tend à montrer que lorsqu'une piscine est contaminée, il est malaisé de supprimer les souches virulentes.

Nous devons cependant noter que Singh et Das (1970) désignent sous le nom de 
Naegleria aerobia les amibes isolées à partir de cas de méningo-encéphalites comme la souche HB 1 de Culbertson.

Ils se basent sur la nature aérobie de cette souche et sur des caractères du kyste. Carter (1970), de son côté, envisage d'appeler $N$. fowleri les amibes qu'il a étudiées en se basant sur l'ultrastructure et l'absence de pore dans les kystes et sur la non viabilité de la plupart de ceux-ci.

Il est néanmoins difficile de suivre ces auteurs et de séparer des parasites dont la morphologie et le comportement sont tellement voisins et dont l'habitat paraît commun. Presque tous les cas de méningo-encéphalites amibiennes ont été diagnostiqués chez des nageurs. Lors de l'épidémie la plus importante et la mieux étudiée par Cervà et ses collaborateurs (1968) à Usti en Tchécoslovaquie, tous les sujets ayant contracté une méningo-encéphalite se sont infectés dans une même piscine.

\section{$2^{\circ}$ Virulence acquise.}

La rareté des cas de méningo-encéphalite conduit naturellement à l'hypothèse d'une virulence acquise. En effet, dans les eaux de piscines comme dans toute collection d'eau, on peut retrouver des amibes du groupe Limax et cependant les cas mortels sont rares, même dans les piscines où les cas se sont répétés. La densité des amibes Limax va de pair avec la pollution de l'eau. Cervà (communication personnelle) qui a bien étudié le problème a montré que le nombre des amibes peut atteindre jusqu'à 5.000 au litre et plus.

Parmi les souches d'amibes Limax qu'il a isolées à partir de l'eau de piscine à Usti et à partir des jardins qui l'entourent, Cervà identifie six souches de Naegleria qui ne sont pas ou peu pathogènes. Pour notre part, sur les 20 souches de piscines anversoises que nous étudions, une souche de Naegleria s'est montrée pathogène par voie intra-cérébrale pour la souris, tout d'abord très peu, ne tuant qu'un animal sur six après quinze jours et puis davantage au point qu'au cinquantième passage presque toutes les souris meurent entre 2 à 5 jours, et on observe des amibes directement dans les broyats du cerveau.

Cependant, cette souche de Naegleria n'a pas un comportement comparable à celui des amibes isolées de cas de méningo-encéphalite. On ne parvient pas à l'entretenir en milieu liquide même en présence de bactéries tuées. Après 4 à 6 repiquages, les amibes s'arrondissent, se lysent et la culture se perd.

$N$. gruberi provenant de cas pathologiques se multiplie axéniquement en milieu liquide, tandis que $N$. gruberi de piscine ne se maintient que sur Agar à $1,5 \%$ en présence de bactéries tuées.

On peut donc exalter la virulence d'une souche par passage sur la souris, mais elle n'acquiert pas les caractères de culture des souches provenant des cas de méningoencéphalite.

D'autre part, nous venons d'isoler une souche $\mathrm{O} 400$ à partir d'agglomérats de matières organiques, qui constituaient de véritables colonies de Naegleria, riches de centaines d'exemplaires. Le prélèvement est fait dans une piscine privée et couverte 
qui a été abandonnée pendant trois semaines au moment où la température extérieure a atteint les $30^{\circ} \mathrm{C}$ pendant une dizaine de jours.

Ces amibes sont inoculées directement dans le cerveau de trois souris et, 24 heures après, une souris est mourante; nous constatons la présence de nombreuses Naegleria qui sont retransmises.

Des souris sont aussi inoculées par voie nasale et une de celles-ci est mourante après huit jours. Il y a de nombreuses amibes dans un poumon hépatisé. Elles sont plus rares dans le cerveau. Cette souche est entretenue par voie nasale.

Les ensemencements en milieu liquide (milieu de Cervà modifié, Willaert 1971) à partir des agglomérats donnent également un bon développement avec amibes et formes flagellées que nous avons pu entretenir régulièrement au cours de dix repiquages à $28^{\circ} \mathrm{C}$, mais non à $37^{\circ} \mathrm{C}$.

Cette observation, toute fortuite, montre que l'on peut donc retrouver même dans un bassin peu fréquenté des amibes qui sont pathogènes.

\section{$3^{\circ}$ Contacts répétés avec les antigènes humains et animaux.}

Il y a cependant d'autres possibilités, notamment le contact des amibes libres avec des antigènes humains et animaux.

Dans les piscines chauffées où les amibes se multiplient dans les filtres, ainsi que Cervà l'a observé, si le nombre des usagers s'élève à certaines heures du jour à des chiffres qui dépassent de loin la capacité d'épuration, l'eau s'enrichit de déchets humains issus de la desquamation de la peau, du pourtour des orifices naturels, des muqueuses nasales.

Ces antigènes ou les acides aminés qui en dérivent peuvent être utilisés comme susbtance nutritive par les amibes. Or, les travaux de Capron et de ses coll. (1968) nous apprennent qu'un parasite ne peut s'implanter dans un organisme que s'il y retrouve des groupes antigéniques communs.

\section{$4^{\circ}$ Le passage d'un individu à l'autre est capable d'exalter la virulence d'une souche.}

De même que l'on a pu constater que la virulence d'Entamoeba histolytica s'exaltait dans les pays où l'épandage est considérable, ainsi dans les piscines surpeuplées les amibes de l'eau passent d'une muqueuse nasale à l'autre et augmente leur pouvoir d'adaptation.

Skocil, Cervà et Serbus (1970) ont trouvé que sur 1000 soldats de 20 à 21 ans, on pouvait isoler par écouvillonnage des muqueuses nasales des Hartmannella chez 49 d'entre eux.

Aux Etats-Unis, Wang et Feldman découvrent 33 porteurs d'amibes Limax sur 2289 personnes. En Angleterre, Pereira et ses collaborateurs (1966) isolent chez des patients présentant des troubles respiratoires un agent pathogène, sous le nom de 
« Ryan virus » qu'Amstrong et Pereira (1967) identifient comme une amibe du genre Hartmannella.

Par ailleurs, Kingston et Warhurst (1969) retrouvent diverses Hartmannella et deux souches de Naegleria dans l'air des chambrettes d'enfants atteints de troubles respiratoires.

Ces quelques observations viennent à l'appui de l'hypothèse qui envisage le passage des amibes d'un sujet à l'autre.

\section{Discussion :}

L'ensemble de ces hypothèses doit, sans doute, être retenu. Pour qu'existent des souches virulentes dans les piscines, il faut que l'eau soit très polluée, que la piscine soit très fréquentée pour assurer un passage rapide des souches d'un sujet à l'autre.

Il faut retenir la leçon d'Usti (Cervà 1968) où 17 cas se sont succédés de 1962 à 1968 ; ce qui montre que lorsqu'une installation est contaminée par une souche virulente, il est nécessaire d'introduire de nombreuses modifications pour faire disparaître les amibes pathogènes.

\section{Bibliographie}

Armstrong (J. A.) et Pereira (M. S.), 1967. - Identification of Ryan virus as in Amoeba of the genus Hartmannella. Brit. Méd. J., 1, 212-214.

Butt (C. G.), Baro (C.) and KNorR (R.W.), 1968. - Naegleria sp. identified in amebic encephalitis. The Am. J. of Clin. Path., 50, 568-574.

Capron (A.), Biguet (J.), Vernes (A.) et Afchain (D.), 1968. - Structure antigénique des helminthes. Aspects immunologiques des relations hôte-parasite. Path. Biol., 16, 121-138.

CARTer (R. F.), 1970. - Description of a Naegleria sp. isolated from two cases of primary amoebic meningo-encephalitis and the experimental pathological changes induced by it. The J. of Pathology, 100, 217-244.

Cerva (L.) et NovaK (K.), 1968. - Amoebic meningo-encephalitis: sixtheen fatalities. Science, $160,92$.

-, - et Culbertson (C. G.), 1968. - An outbreak of acute fatal amebic meningo-encephalitis. Am. J. of Epidemiology, 88, 436-444.

-, Ferdinandova (M.), NovaK (K.) et ZimaK (V.), 1968. - Eitrige Amoben Meningoencephalitis. Tödlich verlaufende Fälle in Nordbohmen. Münch. med. Wschr., 110, 1364-1368.

—, 1969. - Amoebic meningo-encephalitis : axenic culture of Naegleria. Science, 16, 357360.

—, ZINAK (V.) et NovaK (K.), 1969. - Amoebic meningo-encephalitis: a new amoeba isolate. Science, 163, 575-576. 
Chatton (E.) et Lalung-Bonnaire, 1912. - Amoeba limax (Vahlkampfia n. gen.), dans l'intestin humain. Son importance pour l'interprétation des amibes de culture. Bull. Soc. Path. Exot., 5, 135-143.

Culbertson (C. G.), Smith (J. W.), Coken (H. K.) and Minner (J. R.), 1959. - Experimental infection of mice and monkeys by Acanthamoeba. Am. J. Path., 35, 185-197.

-, Ensminger (P. W.) and Overton (W. M.), 1968. - Pathogenic Naegleria sp. Study of a strain isolated from human cerebrospinal fluid. J. Protozoology, 15, 353-363.

Deschiens (R.), 1965. - L'amibiase et l'amibe dysentérique, 697 pp., Paris, Masson.

Fowler (M.) et CARTer (R. F.), 1965. - Acute pyogenic meningitis probably due to Acanthamoeba sp.: a preliminary report. Brit. Med. J., 2, 740-742.

Halpert (B.) and Ashley (J. D.) Jr., 1944. - Amoebic colitis complicated with Abcess of the brain. Arch. Path., 38, 112-114.

Jadin (J. B.), Hermanne (J.), RobiJn (G.) et Willaert (E.), 1971. - Trois cas de méningoencéphalite amibienne primitive en Europe occidentale à Anvers. Bull. Acad. nat. Méd. (Paris), 155, 232-238.

- , - , - - , Van Maercke (Y.) et Stevens (W.), 1971. - Trois cas de méningo-encéphalite amibienne primitive observés à Anvers (Belgique). Ann. Soc. belge Méd. trop., $51,255-266$.

KARTULIS (S.), 1904. - Gehirnabscesse nach dysenterischen leberabscessen. Zbl. Bakt. I. Abt. Oeig., 37, 527-530.

Kingston (D.) and Warhurst (D. C.), 1969. - Isolation of amoebae from the air. J. med. Microbiol., 2, 27-36.

Pereira (M. S.), Marsden (H. B.), Corbitt (G.) et Tobin (J. O. H.), 1966. - Ryan virus : a possible new human pathogen. Brit. med. J., 1, 130-132.

ScHAudinN (F.), 1903. - Untersuchüngen über die Fortpffanzung einiger Rhizopoden (Vorläufige Mitheilung). Hrb. Gesundhamte (Berl.), 19, 547-576.

SiNGH (B. N.) and DAS (S. R.), 1970. - Taxonomy of amoebae placed in the order Amoebida. Kent 1880. Parasit., 56, 319-320.

Skocil (V.), Cervi (L.) and Serbus (C.), 1970. - Epidemiological study of amoebas of the limax group in military communities. First Report., J. Hyg. Epid. Micob. Immunol., 14, 61-66.

Wang (S. S.) et Feldmann (H. A.), 1967. - Isolation of Hartmannella species from human troats. New. Engl. J. Med., 277, 1174-1179.

Wells (R. T.), 1911. - Aerial contamination as a fallacy in the study of amoebic infections by cultural methods. Parasitology, 4, 204-219. 